\title{
Malignant Pertusis: Emphasis on early Leucoreduction
}

Manvi Singh1, Rajiv Suku1, Pankaj.C.Vaidya1, Joseph.L.Mathew1, Meenu Singh1

Department of Pediatrics, Advanced Pediatrics Center, Postgraduate Institute of Medical Education and research, Chandigarh,160012

\section{Introduction}

-Pertussis is a leading cause of respiratory illness leading to significant mortality in developing nations. -There has been a rise in its incidence even in developed countries. The extent of complications is found to be more in early infancy.

-Malignant or critical illness is defined by combination of respiratory failure, pneumonia, hyperleucocytosis, neurological involvement and pulmonary hypertension. It requires management in intensive care settings and may have a mortality of upto $80 \%$.

- We report an infant who presented with malignant pertussis, and underwent leucoreduction by leucopheresis but unfortunately succumbed to the illness. We also present a brief review of the utility and modes of leucoreduction.

\section{Case report}

-An 8 weeks old developmentally normal baby had received birth dose of Bacillus Calmette Guerin and Oral Polio Vaccine.

- Child presented with acute onset of respiratory distress in the form of paroxysms of cough with fever and lethargy.

-Investigations revealed total leucocyte count of

114000 cells per cubic millimeter.

-A clinical possibility of pertussis was kept.

Azithromycin was added.Throat swab was sent for pertussis PCR came positive.

References

1. Oñoro G, Salido AG, Martínez IM, Cabeza B, Gillén M, de Azagra AM. Leukoreduction in patients with severe pertussis with hyperleukocytosis. Pediatr Infect Dis J. 2012 Aug;31(8):873-6.

2. Sloan SR, Andrzejewski C, Aqui NA, Kiss JE, Krause PJ, Park YA. Role of therapeutic apheresis in infectious and inflammatory diseases: Current knowledge and unanswered questions. J Clin Apheresis. 2015 Oct;30(5):259-64.

3. Kuperman A, Hoffmann Y, Glikman D, Dabbah H, Zonis Z. Severe pertussis and hyperleukocytosis: is it time to change for exchange? Transfusion (Paris). 2014 Jun;54(6):1630-3

4. Berger JT, Carcillo JA, Shanley TP, Wessel DL, Clark A, Holubkov R, et al. Critical Pertussis Illness in Children, A Multicenter Prospective Cohort Study. Pediatr Crit Care Med J Soc Crit Care Med World Fed Pediatr Intensive Crit Care Soc [Internet]. 2013 May [cited 2018 Feb 7];14(4). Available from: https://www.ncbi.nlm.nih.gov/pmc/articles/PMC3885763/

\begin{abstract}
-The child had persistent tachypnoea, tachycardia in tune of 200, with high TLC, and hence the diagnosis of malignant / critical pertussis was kept.

-Leucoreduction by leucopheresis which was done with documented decrease in TLC.

-Three hours post procedure, baby developed repeated bouts of cough with increased work of breathing and

saturation dips, was intubated for the same. However his clinical condition deteriorated with development of shock and the child succumbed to the illness.
\end{abstract}



Chest X-ray of the index case

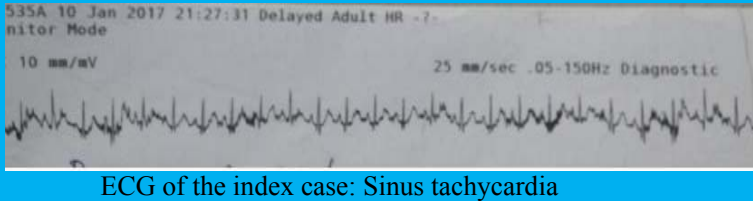

ECG of the index case: Sinus tachycardia

\section{Discussion}

perissis is a serious illness caused by Bordetella ussis and is a leading cause of mortality under 6 months of age, particularly in those babies who are unimmunized.

Infection is transmitted parents or siblings whose unity has waned.

Mertusis carries significant mortality. Hyperleucocytosis is a known complication. It is defined as TLC $>100000$ per cubic $\mathrm{mm}$. It can be asymptomatic or symptomatic in the form of dyspnoea, tachypnoea, hypoxemia and fever. Symptomatic hyperleucocytosis had been observed even in those with counts around $30,000 / \mathrm{ml}$. Vital parameters that indicate critical pertussis include tachycardia exceeding 170/min, tachypnoea more an $70 / \min .3$

The mechanism of critical pertusis:

$>$ Increased blood viscosity due to the elevated counts leading to decreased organ perfusion, pulmonary thrombosis and pulmonary artery hypertension.

$>$ Endothelial damage due to the direct ect of the toxin
The most dreaded complications are intracrania hemorrhage and pulmonary insufficiency leading to hypoxemia, particularly in small infants.

The methods that we can adopt for leucoreduction are an exchange transfusion or a leucopheresis. The choice between the two is uncertain due to the lack of trials.

-Leucopheresis can be tried if there is adequate aphaeresi apparatus with trained staff available.

Exchange transfusion has also been shown to be effective in preventing mortality in cases of malignant pertusis and is indicated for small infants with pertussis with a total count of more than 1 lakh, between 70,000-100000 if with respiratory failure, if more than 50,000 with evidence of pulmonary artery hypertension. This improves the oxygenation by improving the gas exchange, and prevents urther myocardial injury.

\section{Conclusion}

Malignant pertussis is a life threatening disease in infancy. Strict immunization practices, early recognition of critical pertussis and timely leucoreduction are the key for survival.

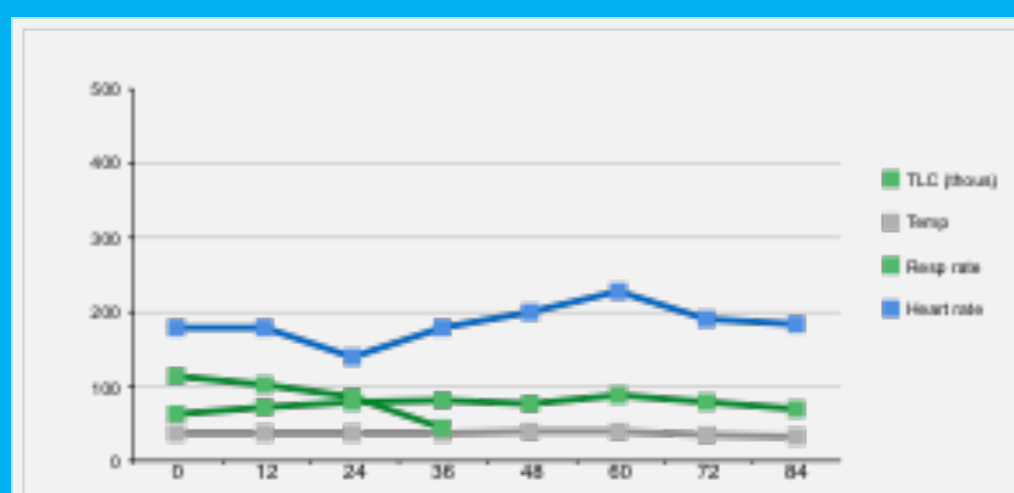

Vitals chart: Despite falling counts after leucopheresis, the heart rate continued to increase indicating an irreversible insult caused by the hyperleucocytosis. 